\title{
Effects of low- and high-volume resistance exercise on postprandial lipaemia
}

\author{
Andreas Zafeiridis ${ }^{1,3}$, Evagelia Goloi ${ }^{1}$, Anatoli Petridou ${ }^{2}$, Konstantina Dipla ${ }^{1}$, \\ Vassilis Mougios ${ }^{2}$ and Spiros Kellis ${ }^{1}$ \\ ${ }^{1}$ Department of Physical Education and Sport Science, Sports Performance and Coaching Laboratory, Aristotelio University of \\ Thessaloniki, Thessaloniki, Greece \\ ${ }^{2}$ Department of Physical Education and Sport Science, Sport Hygiene and Nutrition Laboratory, Aristotelio University of \\ Thessaloniki, Thessaloniki, Greece \\ ${ }^{3}$ Department of Physical Education and Sport Sciences, Serres T.E.F.A.A., Serres, Greece
}

(Received 8 March 2006 - Revised 31 July 2006 - Accepted 9 October 2006)

\begin{abstract}
Postprandial lipaemia (PL) is associated with the metabolic syndrome, CVD and endothelial dysfunction. Aerobic exercise has been shown to reduce PL. Although resistance exercise is recommended for the improvement of the quality of life, management of body weight and prevention of several disorders, its effect on PL has received little attention. The present study examined the effects of low-volume resistance exercise (LVRE) and high-volume resistance exercise (HVRE) on PL. Ten healthy young men performed three trials, each conducted over $2 \mathrm{~d}$. On the afternoon of day 1, they either refrained from exercise (control), performed LVRE (two sets of eight exercises, twelve repetitions at twelve repetitions maximum (RM) in each set; energy expenditure $0.76 \mathrm{MJ}$ ), or performed HVRE (four sets of eight exercises, twelve repetitions at 12RM in each set; energy expenditure $1.40 \mathrm{MJ}$ ). On the morning of day 2 they consumed a meal containing $67 \mathrm{~kJ} / \mathrm{kg}$ body weight, of which $65 \%$ energy was from fat. Blood samples were obtained in the fasted state and for $6 \mathrm{~h}$ postprandially. The total area under the TAG curve (AUC; mmol/l $\times \mathrm{h})$ was lower $(P<0.05)$ in HVRE (8.76 (SD 3.20)) and LVRE (9.29 (SD 3.64)) compared with control (11.60 (SD 4.35)). The incremental AUC was lower in HVRE compared with control (3.07 (SD 2.53) v. 5.58 (SD 3.72)), but not different between LVRE (3.86 (SD 2.29)) and control. In conclusion, resistance exercise of $1.40 \mathrm{MJ}$ (four sets - eight exercises - twelve RM) or 0.76 MJ (two sets - eight exercises - twelve RM) before a highfat meal reduces the total postprandial lipaemic response.
\end{abstract}

Postprandial lipaemia: Triacylglycerols: Weight lifting: Resistance exercise: Energy expenditure

Postprandial lipaemia (PL) is associated with the metabolic syndrome, CVD, endothelial dysfunction and atherogenesis (Gotto, 1998). Thus, reducing PL may lower the risk for the development of these disorders. Acute aerobic exercise about $15-18 \mathrm{~h}$ before a high-fat meal attenuates PL (Tsetsonis \& Hardman, 1996; Tsetsonis et al. 1997; Malkova et al. 1999; Gill et al. 2001; Gill et al. 2002a). The energy expended during aerobic exercise is a key determinant of the exercise-induced attenuation of PL (Gill et al. 2002a; Gill \& Hardman, 2003).

Resistance exercise is recommended by health associations for the improvement of the quality of life, management of body weight and prevention of several disorders (Jakicic et al. 2001; Kraemer et al. 2002; Kohrt et al. 2004; Sigal et al. 2006). To date three studies have examined the delayed (after 14-16h) effects of resistance exercise on PL and have reported either reduction (Petitt et al. 2003) or no change (Burns et al. 2005; Shannon et al. 2005). Another study examined the effect after $1 \mathrm{~h}$ of resistance exercise and reported an increase in PL (Burns et al. 2006). It should be highlighted that Shannon et al. (2005) and Burns et al. (2006) used different experimental approaches from that of Petitt et al.
(2003) and Burns et al. (2005). Thus, the effects of resistance exercise on PL are not clear.

According to the American College of Sports Medicine recommendation for novice to intermediate trainees (Kraemer et al. 2002), the amount of resistance exercise in the studies of Petitt et al. (2003) and Burns et al. (2005), three to four sets of ten to eleven exercises for ten repetitions, represents a high target for well-motivated individuals of the general population. Since exercise dose is an important consideration when designing health-related exercise programmes, it is essential to know whether lower volumes of resistance exercise, which may be more attainable by the general public, mitigate PL. The only study that examined the effects of different volumes of resistance exercise on PL reported no dose-response relationship (Shannon et al. 2005). However, the authors investigated the dose-response issue while counterbalancing the energy deficit of exercise. That is, after performing resistance exercise (one, three or five sets), the subjects consumed proportionally more energy and fat compared with the control trial (reaching over two-fold) on the night before the fat-tolerance test. This may have increased

Abbreviations: AUC, area under the curve; HOMA-IR, homeostasis model assessment of relative insulin resistance; HVRE, high-volume resistance exercise; LVRE, low-volume resistance exercise; PL, postprandial lipaemia; RM, repetitions maximum.

* Corresponding author: Dr Andreas Zafeiridis, fax + 30 2310992186, email zafeiridis@medscape.com 
the likelihood of dietary modification as a confounding factor masking the effect of resistance exercise on the postprandial lipaemic response. It would be of interest to examine the effects of various doses of resistance exercise on PL without abolishing the exercise-related manifestations, which include energy deficit as well as physiological, metabolic and hormonal perturbations. Furthermore, the authors of the same study (Shannon et al. 2005) based their findings on a mixed sample of males and females, although they reported a different postprandial lipaemic response to resistance exercise between the sexes.

Based on the contradictory findings described, the delayed effects of resistance exercise on PL require further investigation. In addition, no study has investigated the effects of various doses of resistance exercise on PL in men, while controlling for dietary intake on the day before the fat-tolerance test. With these issues in mind, the present study was designed to investigate the effects of prior high-volume resistance exercise (HVRE) and lowvolume resistance exercise (LVRE) on PL.

\section{Subjects and methods}

\section{Subjects}

Ten healthy young men (age 24.6 (SD 1.5) years; body weight 77.8 (SD 8.2) kg; height 1.79 (SD 0.04) m; BMI 24.2 (SD 2.2) $\mathrm{kg} / \mathrm{m}^{2}$; body fat $17 \cdot 1(\mathrm{SD} 3 \cdot 8) \%$ ) with recreational experience in weight lifting (two to three times/week for over 1 year) volunteered to participate in the study and signed an informed consent form. All subjects completed a medical history questionnaire and were included in the study if they were free of cardiac, respiratory and metabolic diseases, and if they were not taking any medication or dietary supplements. The study was approved by the institutional ethics committee.

\section{Study design}

All subjects performed in random order three trials spaced at least 1 week apart, each trial conducted over $2 \mathrm{~d}$. On the afternoon of day 1 they either refrained from exercise (control), performed LVRE, or performed HVRE. On the morning of day 2 , after $12 \mathrm{~h}$ of fasting, the subjects consumed a high-fat meal. The meal was administered approximately $16 \mathrm{~h}$ after the end of the resistance exercise trials. Blood samples were obtained at baseline and at $0 \cdot 5,1,2,3,4,5$ and $6 \mathrm{~h}$ after the meal for the measurement of plasma TAG, glucose and insulin. The study design is depicted in Fig. 1.

\section{Preparation for the trials}

The subjects were asked to record their diet for $2 \mathrm{~d}$ before the initiation of the study. Then they were asked to replicate this diet during the $2 \mathrm{~d}$ before each test meal and record their actual diet. Furthermore, subjects were instructed to abstain from alcohol ingestion and vigorous physical activity (except for the experimental exercise) at least $2 \mathrm{~d}$ before each test meal, and from caffeine consumption at least $1 \mathrm{~d}$ before each test meal.

\section{Resistance exercise protocols}

On the two occasions that each subject performed resistance exercise, they reported to the laboratory at 15.00-16.00 hours on day 1. A portable gas analyser (VO2000; MedGraphics, St Paul, MN, USA) was calibrated and the subjects wore the mask for the measurement of energy expenditure throughout the exercise session. The two experimental resistance exercise protocols consisted of eight exercises that were performed in the following order: leg extension, bench press, leg press, latissimus dorsi pull-down, calf-raise, biceps curl, triceps extension, and sit-up. The sit-ups were performed until failure, whereas all other exercises consisted of twelve repetitions at twelve repetitions maximum (RM) in each set. The load corresponding to twelve RM had been determined $7 \mathrm{~d}$ before the first trial.

In LVRE the subjects performed two sets and in HVRE four sets in each exercise station before proceeding to the next one. The selection of exercise parameters (sets and repetitions) was based on the following considerations: (i) repetitions should not exceed the upper limit of twelve RM set by the American College of Sport Medicine for improvement of muscular strength for novice to intermediate-level trainees; (ii) one protocol should approximate the energy expenditure in the study by Petitt et al. (2003) and the other should have half that energy expenditure; (iii) four sets elicit maximal strength

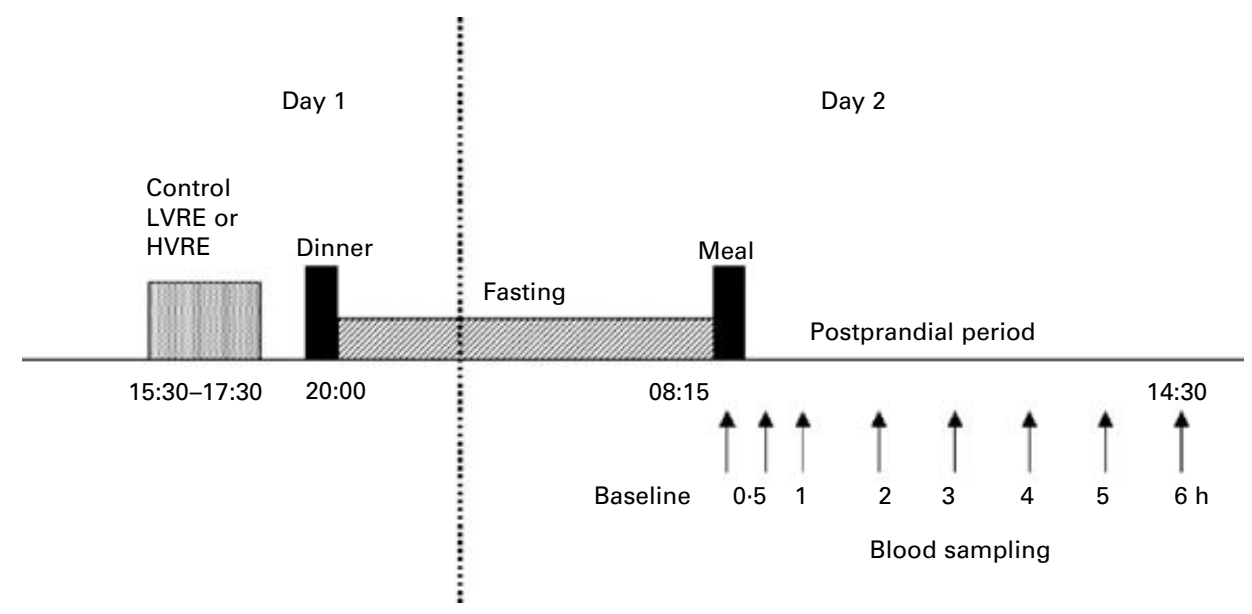

Fig. 1. Study design. LVRE, low-volume resistance exercise; HVRE, high-volume resistance exercise. 
gains in trained and untrained individuals (Rhea et al. 2003), and most recreationally active individuals perform one to four sets of eight to twelve RM in their resistance exercise programmes.

In both protocols the resting period was $1.5 \mathrm{~min}$ between sets and 2 min between exercises. If, during the execution of a set before the final one, a subject was unable to complete twelve repetitions, the weight was reduced by $5 \mathrm{~kg}$ in the next set(s). The total duration of the LVRE trial was 39 (SD 1) min and that of the HVRE trial 79 (SD 1) min. Soreness was assessed at $48 \mathrm{~h}$ after the resistance exercise trials by using a scale of perceived pain from 0 (no pain) to 10 (very very painful).

\section{Experimental meal}

On arrival of the subjects at the laboratory at 08.00 hours on day 2 , a catheter was inserted into a forearm vein for serial blood collections. The subjects were weighed, rested for $15 \mathrm{~min}$ and provided a $5 \mathrm{ml}$ blood sample for baseline measurements. Then, each subject consumed a meal consisting of milk, bread, butter, mayonnaise, cheese, salami and chocolate. The meal contained $5.2(\mathrm{SD} 0.5) \mathrm{MJ}(67 \mathrm{~kJ} / \mathrm{kg}$ body weight); energy derived was $65 \%$ from fat, $21 \%$ from carbohydrate and $14 \%$ from protein. After the meal the subjects were not allowed to eat or exercise for $6 \mathrm{~h}$ but were given free access to water, the volume of which was recorded during the first trial and was administered in the subsequent trials. Blood samples were drawn at $0.5,1,2,3,4,5$ and $6 \mathrm{~h}$ after meal consumption as described for the baseline sample. No subject reported nausea or other gastrointestinal discomfort.

\section{Anthropometric measurements and analytical procedures}

Body weight was measured to the nearest $0.1 \mathrm{~kg}$ by an electronic balance (Seca, Hamburg, Germany) and height was measured to the nearest $1 \mathrm{~cm}$ by a stadiometer fixed to the balance. Percentage body fat was estimated from skinfold measurement at triceps and subscapular sites (Slaughter et al. 1988). Food records were analysed as described (Kolifa et al. 2004). Blood was collected in tubes containing EDTA and was immediately centrifuged at $1500 \mathrm{~g}$ at $4^{\circ} \mathrm{C}$ for $5 \mathrm{~min}$. Plasma was removed, separated into samples and stored at $-80^{\circ} \mathrm{C}$ for later analysis of TAG, glucose and insulin. TAG and glucose were measured by enzymic photometric methods using reagent kits from BEST (Athens, Greece). Insulin was analysed by enzyme immunoassay using a kit from DRG (Marburg, Germany). All samples were analysed on a single day for each parameter to eliminate inter-assay variability. The intra-assay $\mathrm{CV}$ were $1.8 \%$ for TAG, $1.8 \%$ for glucose and $3.8 \%$ for insulin.

\section{Statistical analysis and calculations}

All data are presented as mean values and standard deviations and were analysed by using Statistica version 6.0 (StatSoft Inc., Tulsa, OK, USA). A two-way ANOVA (protocol $\times$ time) with repeated measures on both factors was used to analyse the TAG, glucose and insulin concentrations. Areas under the curve (AUC), calculated as previously described (Kolifa et al. 2004), were used as summary measures of the TAG, glucose and insulin responses. In addition, we calculated the incremental
TAG AUC, which eliminates the contribution of the fasting TAG concentration to the postprandial lipaemic response. The insulin sensitivity index was calculated by the HOMA2 calculator (version 2.2; Diabetes Trials Unit, University of Oxford, Oxford, UK) using the baseline values of glucose and insulin. One-way ANOVA with repeated measures was used to compare the AUC for each parameter, homeostasis model assessment of relative insulin resistance (HOMA-IR) and the dietary data. Where appropriate, ANOVA tests were followed by Scheffé pairwise comparisons to identify significantly different means. All statistical tests were performed with a two-tailed hypothesis, accepting $P<0.05$ as significant.

\section{Results}

The gross energy expenditure in the LVRE and HVRE protocols was 0.76 (SD 0.09) and 1.40 (SD 0.17) MJ, respectively $(P<0.001)$. At $48 \mathrm{~h}$ after the resistance exercise protocols the subjects reported none to mild overall muscle soreness, scoring 1.0 (SD 0.9) points after LVRE and 1.3 (SD 0.7) points after HVRE. Energy and macronutrient intake over the $2 \mathrm{~d}$ before each fat-tolerance test were not different among trials (Table 1).

Fig. 2 shows the plasma TAG concentrations before the high-fat meal and during $6 \mathrm{~h}$ after its consumption in the control, LVRE and HVRE trials. Two-way ANOVA revealed significant main effects of protocol and time on TAG $(P<0 \cdot 001)$. Post hoc analysis within main effects showed that TAG values were significantly lower in HVRE $(P=0.002)$ and LVRE $(P=0.006)$ compared with control, and TAG concentrations increased significantly $2-6 \mathrm{~h}$ postprandially compared with baseline $(P<0 \cdot 01)$.

One-way ANOVA revealed a significant effect of protocol on the total and incremental TAG AUC $(P=0.002$ and 0.036, respectively; Fig. 3). Pairwise comparisons showed that the total TAG AUC was lower in LVRE (9.29 (SD 3.64) mmol/l $\times \mathrm{h})$ and HVRE $(8.76(\mathrm{SD} 3.20) \mathrm{mmol} / \mathrm{l} \times \mathrm{h})$ compared with control $(11.60(\mathrm{SD} 4.35) \mathrm{mmol} / \mathrm{l} \times \mathrm{h})$ by $20 \%(P=0.017)$ and $24 \%(P=0.004)$, respectively. The effect sizes (difference of means divided by the SD of control) were -0.53 for LVRE and -0.65 for HVRE. Eight of the ten subjects demonstrated lower total TAG AUC values in LVRE and HVRE compared with control. The incremental TAG AUC was lower in HVRE (3.07 (SD 2.53) $\mathrm{mmol} / \mathrm{l} \times \mathrm{h})$ compared with control $(5.58$ $(\mathrm{SD} 3.72) \mathrm{mmol} / \mathrm{l} \times \mathrm{h})$ by $45 \%(P=0.041)$, but not significantly different between LVRE (3.86 (SD 2.29) $\mathrm{mmol} / \mathrm{l} \times \mathrm{h})$ and control $(P=0 \cdot 195)$. The effect sizes for the incremental

Table 1. Dietary intake over the $2 \mathrm{~d}$ before the fat-tolerance test in each trial (n 10)

(Mean values and standard deviations)

\begin{tabular}{|c|c|c|c|c|c|c|}
\hline \multirow{2}{*}{$\begin{array}{l}\text { Protocol... } \\
\text { Variable }\end{array}$} & \multicolumn{2}{|c|}{ Control } & \multicolumn{2}{|c|}{ LVRE } & \multicolumn{2}{|c|}{ HVRE } \\
\hline & Mean & $\mathrm{SD}$ & Mean & $\mathrm{SD}$ & Mean & SD \\
\hline Daily energy intake (MJ) & $12 \cdot 2$ & 2.4 & $12 \cdot 3$ & $2 \cdot 3$ & $12 \cdot 4$ & $2 \cdot 6$ \\
\hline Carbohydrate (\% energy) & 43 & 6 & 43 & 9 & 45 & 9 \\
\hline Fat (\% energy) & 40 & 5 & 41 & 8 & 39 & 8 \\
\hline Protein (\% energy) & 17 & 2 & 16 & 3 & 16 & 2 \\
\hline
\end{tabular}

LVRE, low-volume resistance exercise; HVRE, high-volume resistance exercise. 


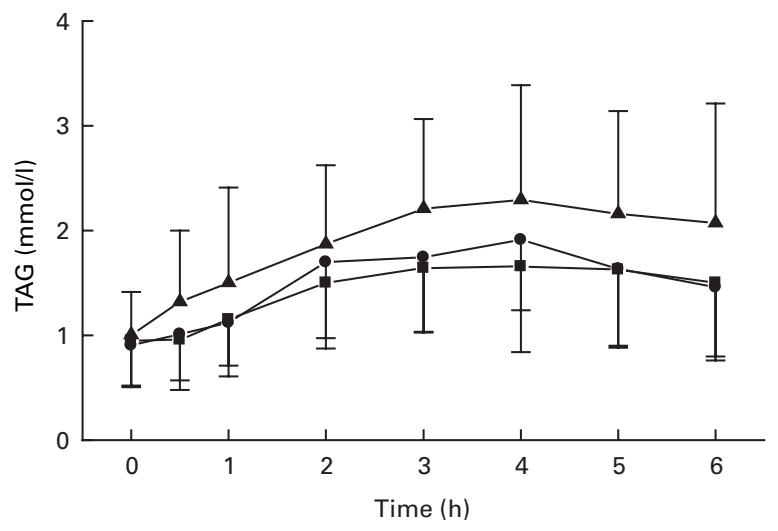

Fig. 2. TAG concentrations in the fasted state and for $6 \mathrm{~h}$ after consumption of a high-fat meal in the control $(\mathbf{\Delta})$, low-volume resistance exercise (LVRE; $\boldsymbol{\bullet})$, and high-volume resistance exercise (HVRE; $\boldsymbol{\square}$ ) trials. Values are means ( $n$ 10), with standard deviations represented by vertical bars. Two-way ANOVA indicated main effects of protocol and time $(P<0.001)$. Scheffé tests within main effects revealed that HVRE and LVRE were lower than control $(P<0.01)$, and 2-6h were higher than $0 \mathrm{~h}(P<0.01)$.

TAG AUC were -0.46 for LVRE and -0.68 for HVRE. Given the classification of effect sizes about 0.2 as small, 0.5 as moderate, and 0.8 as large, the effects of the two resistance exercise protocols on PL should be considered as moderate to high. There were no significant differences between LVRE and HVRE in total or incremental TAG AUC.

The plasma glucose and insulin responses in the three trials are presented in Fig. 4. Two-way ANOVA indicated only a significant main effect of time on both parameters $(P<0.001)$. Scheffé follow-up analysis showed that glucose increased at $0.5 \mathrm{~h}(P<0.001 ;$ Fig. $4(\mathrm{~A}))$ and insulin increased at $0 \cdot 5,1$ and $2 \mathrm{~h}$ postprandially $(P<0 \cdot 001$; Fig. $4(\mathrm{~B}))$. The total glucose and insulin AUC were not significantly different among trials (Fig. 4 (C)).

The HOMA-IR index was not significantly different among the control (1.4 (SD 0.4)), LVRE (1.4 (SD 0.5)) and HVRE (1.2 (SD 0.4)) trials $(P=0.441)$.

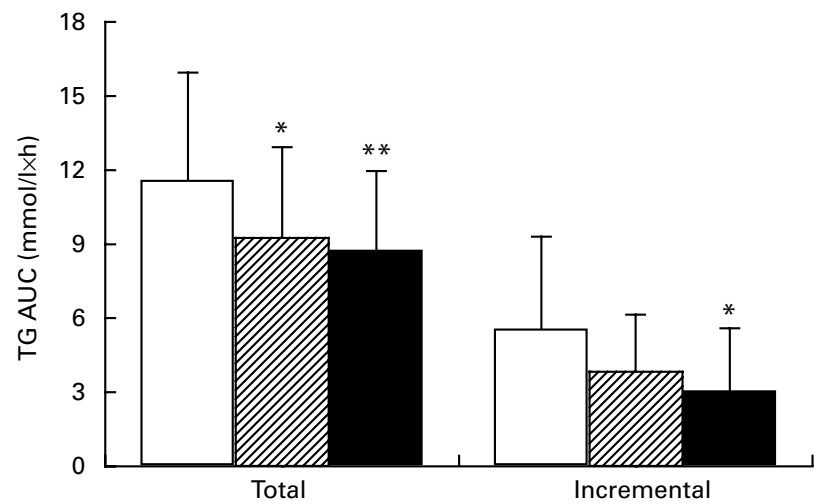

Fig. 3. Total and incremental TAG area under the curve (AUC) for $6 \mathrm{~h}$ after consumption of a high-fat meal in control $(\square)$, low-volume resistance exercise (圆), and high-volume resistance exercise (ם). Values are means $(n 10)$, with standard deviations represented by vertical bars. One-way ANOVA showed an effect of protocol on the total and incremental TAG AUC $(P=0.002$ and $P=0.036$, respectively). Mean values were significantly different from control (Scheffé follow-up test): * $P<0.05,{ }^{\star \star} P<0.01$.
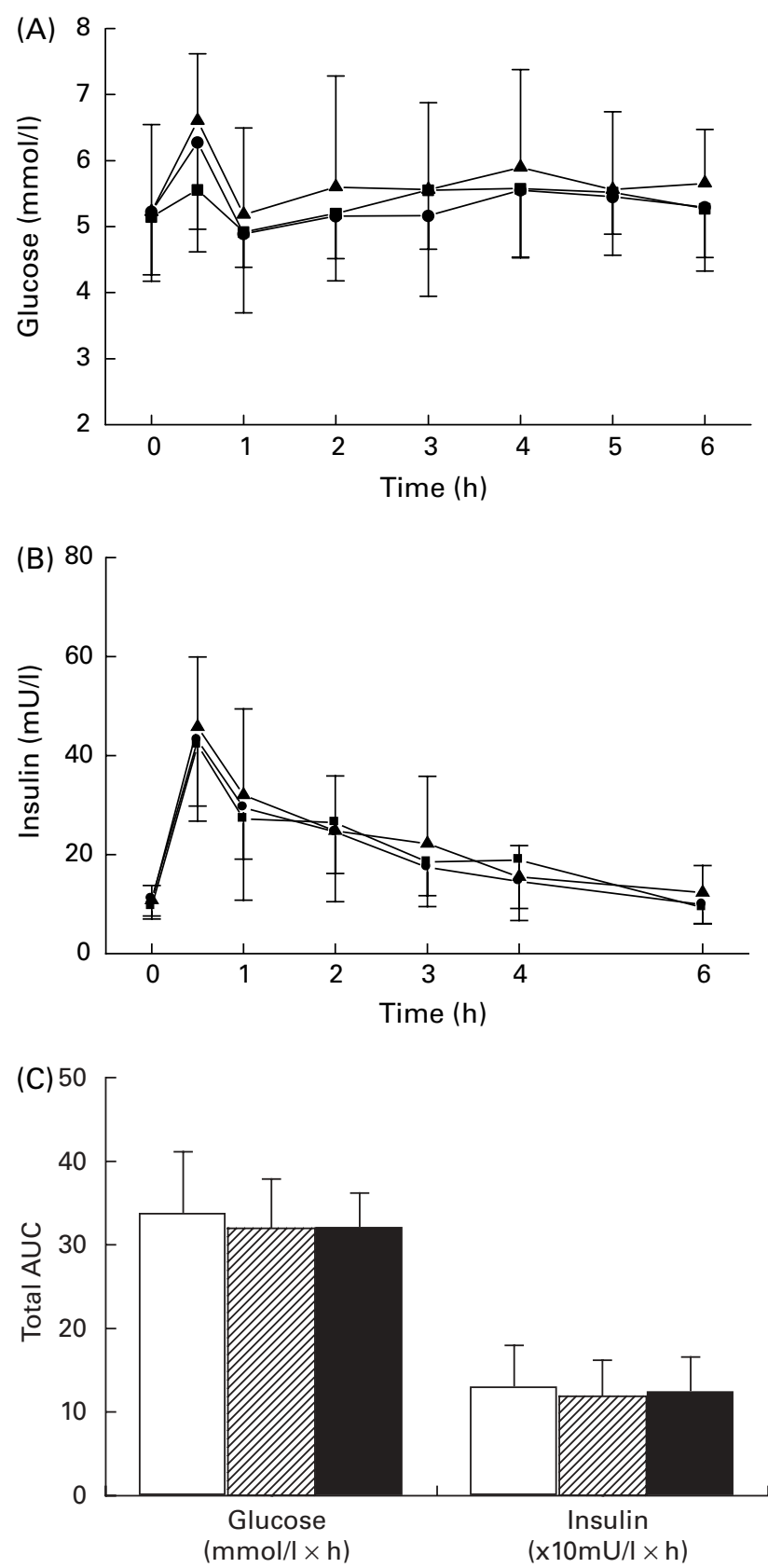

Fig. 4. Glucose (A) and insulin (B) concentrations in the fasted state and for $6 \mathrm{~h}$ after consumption of a high-fat meal in control $(\mathbf{\Lambda})$, low-volume resistance exercise (LVRE; $\bullet$ ) and high-volume resistance exercise (HVRE; $\mathbf{\square})$. Twoway ANOVA indicated only a main effect of time on glucose and insulin $(P<0.001)$. Scheffé analysis within the main effect of time showed that glucose increased at $0.5 \mathrm{~h}(P<0.001)$ and insulin increased at $0.5-2 \mathrm{~h}$ postprandially $(P<0.001)$. Total glucose and insulin area under the curve (AUC) (C) were not different among trials: control $(\square)$; LVRE (ש); HVRE (ם). Values are means $(n 10)$, with standard deviations represented by vertical bars.

\section{Discussion}

The major finding of the present study was that resistance exercise of either 1.40 or $0.76 \mathrm{MJ}$, performed approximately $16 \mathrm{~h}$ before a high-fat meal, lowered the total postprandial lipaemic response. The present results suggest that resistance exercise may be successful in reducing PL at doses that are 
lower - and more attainable - than the $1.70 \mathrm{MJ}$ originally documented by Petitt et al. (2003). Thus, the present study confirms that resistance exercise is effective at a much lower dose than that of continuous aerobic exercise (2.0-2.3 MJ) (Tsetsonis et al. 1997; Miyashita et al. 2006). On the other hand, intermittent aerobic exercise of only 1.0 MJ has been found to reduce PL (Altena et al. 2004). Collectively, the present results along with those of Petitt et al. (2003) and Altena et al. (2004) suggest that energy expenditure may not be the only factor determining the effect of exercise on PL; exercise type (aerobic $v$. resistance) and method (continuous $v$. intermittent) may also be essential.

The present results are in contrast to the findings of Shannon et al. (2005) and Burns et al. (2005), that resistance exercise of 0.57 to $2.58 \mathrm{MJ}$ energy expenditure did not attenuate PL. However, as pointed out earlier, Shannon et al. (2005) counterbalanced the energy deficit of exercise by increasing (over twofold) energy intake before the fat-tolerance test, unlike the other studies that examined the effects of aerobic or resistance exercise on PL. Thus, the difference from the present results may be attributed to the different dietary manipulation and energy status of the subjects before the fat-tolerance test. Although the energy deficit may affect PL, it should be considered as an exercise-specific feature analogous to physiological, hormonal and metabolic responses. Certainly, it is essential to understand whether the energy status before a high-fat meal alters the effect of resistance exercise on PL. Moreover, Shannon et al. (2005) did not address the different responses of males and females despite a significant sex effect on PL, which may have contributed to the discrepant results. The reason for the different results between the present study and Burns et al. (2005) is not apparent. Both studies used young males and relatively similar protocols. The only difference was that Burns et al. (2005) used subjects not regularly involved in resistance training. We used subjects with recreational experience in weight lifting to ensure that they would complete the heavy resistance exercise without extensive muscle damage (see muscle soreness scores), since muscle damage has been suggested to interfere with PL. However, we did not use subjects with large weight-lifting experience to make the results applicable to a larger population. The issue that muscle damage may alter PL has been addressed by Petitt et al. (2003) and Burns et al. (2005, 2006). In fact, the latter two studies by Burns et al. have implicated muscle damage in their inability to document a reducing effect of resistance exercise on PL (through an impaired uptake of TAG from the circulation). Thus, the similarity of the present findings with those of Petitt et al. (2003) and the discrepancy with those of Burns et al. (2005) may be attributed to greater muscle damage in the latter study. However, the effect of muscle damage on PL is speculative and requires investigation.

It is difficult to explain the quite similar attenuation of PL after aerobic exercise and resistance exercise of lower energy expenditure. However, it should be pointed out that the metabolic stress, as indicated by muscle fibre type recruitment, muscle TAG and glycogen use, cardiovascular and hormonal responses, rating of perceived exertion, excess post-exercise $\mathrm{O}_{2}$ consumption, metabolite accumulation, and protein turnover, appears to be higher after resistance than aerobic exercise (Vanhelder et al. 1985; Burlerson et al. 1998; Coyle, 2000). In addition, the intermittent nature of resistance exercise may contribute to its higher efficiency compared with aerobic exercise, since Altena et al. (2004) reported that intermittent exercise - three $10 \mathrm{~min}$ bouts with no meal in between - was more effective than continuous exercise of similar energy expenditure in lowering PL. Also, a recent study reported reductions in fasting TAG and insulin, as well as in the postprandial incremental TAG and insulin AUC after intermittent games activity but not after continuous exercise (Barrett et al. 2006). In contrast, other studies have not documented that intermittent exercise is more advantageous in reducing PL (Gill et al. 1998; Murphy et al. 2000; Miyashita et al. 2006). It should be pointed out that the latter studies included meals between intermittent exercise bouts, which may have confounded the postprandial lipaemic response, as suggested by Altena et al. (2004), and masked the additive effects of short exercise bouts. Furthermore, those studies employed high rest:exercise ratios (ranging from $6 \cdot 5: 1$ to $24: 1$ ), which may have reduced the overall stress of exercise, as opposed to a 2:1 ratio in Altena et al. (2004).

The mechanisms responsible for the exercise-induced attenuation of PL observed in the present study are not readily apparent. An attractive explanation for the attenuated postprandial lipaemic response is related to the hypothesis of exerciseinduced depletion of intramuscular energy stores and their subsequent replenishment (Gill \& Hardman, 2003). Increased lipid oxidation, which persists during recovery, may serve to replenish the muscle TAG and/or glycogen stores utilised during exercise (Gill \& Hardman, 2003; Kimber et al. 2003). Indeed, resistance exercise reduced glycogen and muscle TAG by 23 to $30 \%$ (Essen-Gustavsson \& Tesch, 1990; Koopman et al. 2006) and increased energy expenditure and fat oxidation during 10-24 h after resistance exercise (Melby et al. 1993; Osterberg \& Melby, 2000; Jamurtas et al. 2004). On the other hand, Melanson et al. (2002) reported that $24 \mathrm{~h}$ fat oxidation was unaffected by either resistance or aerobic exercise. However, evidence questions the influence of muscle lipid repletion on PL. The attenuation of PL after aerobic exercise could not be explained by the leg uptake of TAG (Malkova et al. 2000), and the postprandial lipaemic response was independent of the relative contribution of fat and carbohydrate used during aerobic exercise (Malkova et al. 1999). Concerning resistance exercise, muscle lipid stores were replenished within $2 \mathrm{~h}$ post-exercise (Koopman et al. 2006), although glycogen stores remained depleted. Thus, the role of muscle lipid replenishment in the mitigation of PL after resistance exercise may be challenged, but that of glycogen cannot be excluded.

It has also been suggested that the reduced PL following exercise is most probably related to increased TAG clearance from the circulation (mediated by LPL) and/or reduced hepatic TAG secretion (Gill \& Hardman, 2003). This is in line with the increased plasma and muscle LPL activity for up to $24 \mathrm{~h}$ after exercise (Lithell et al. 1981, 1984; Ferguson et al. 1998; Hamilton et al. 1998), the increased TAG clearance in the postprandial state $16 \mathrm{~h}$ after exercise (Malkova et al. 2000) and the correlation of exercise-induced changes in plasma LPL activity with changes in PL (Gill et al. 2003). However, all these findings are limited to aerobic exercise.

The postprandial insulin and glucose responses were not different among the three trials, in accordance with Petitt et al. (2003), Burns et al. (2005) and Shannon et al. (2005). In addition, the insulin sensitivity index was not affected by resistance exercise, in agreement with Shannon et al. (2005). These 
findings show that insulin sensitivity did not contribute to the reduced PL after resistance exercise, in line with evidence that insulin sensitivity does not mediate the reduced PL after aerobic exercise (Gill et al. 2002b). Studies on insulin sensitivity in healthy subjects after $12-24 \mathrm{~h}$ of resistance exercise have provided equivocal results. Fluckey et al. (1994) reported reduced insulin with no change in glucose, while Fenicchia et al. (2004) and Chapman et al. (2002) found no changes in glucose, insulin or insulin sensitivity. A recent study that used an insulin tolerance test observed no change in basal glucose and insulin, and increase in insulin sensitivity $24 \mathrm{~h}$ after resistance exercise (Koopman et al. 2005). However, in the same study the estimation of insulin sensitivity by the HOMA-IR index did no show an effect of resistance exercise (as in the present study). Thus, different methods of estimating insulin sensitivity may provide different outcomes.

In conclusion, HVRE (four sets of eight exercises at twelve $\mathrm{RM} ; 1.40 \mathrm{MJ}$ ) and resistance exercise of lower volume (two sets of eight exercises at twelve RM; $0.76 \mathrm{MJ}$ ) performed $16 \mathrm{~h}$ before a high-fat meal reduced PL. Our findings extend the recognised beneficial effects of resistance exercise on health and wellbeing (Jakicic et al. 2001; Kohrt et al. 2004; Sigal et al. 2006), given the association of PL with the metabolic syndrome, endothelial dysfunction and CVD, and may apply to individuals who have difficulty in performing weight-bearing aerobic activities. In light of this and previous studies with resistance exercise, it appears that its effect on PL may depend on the energy status and the training status. It is unknown why resistance exercise of lower energy expenditure than aerobic exercise produces comparable postprandial lipaemic responses. It is possible that this is related to the higher metabolic stress of resistance exercise and to its intermittent nature. Therefore, type and method, as well as energy expenditure, may be important determinants of the exercise-induced reduction in PL.

\section{References}

Altena TS, Michaelson JL, Ball SD \& Thomas TR (2004) Single sessions of intermittent and continuous exercise and postprandial lipemia. Med Sci Sports Exerc 36, 1364-1371.

Barrett LA, Morris JG, Stensel DJ \& Nevill ME (2006) Effects of intermittent games activity on postprandial lipemia in young adults. Med Sci Sports Exerc 38, 1282-1287.

Burlerson MA Jr, O'Bryant HS, Stone MH, Collins MA \& Triplett-McBride T (1998) Effect of weight training exercise and treadmill exercise on post-exercise oxygen consumption. Med Sci Sports Exerc 30, 518-522.

Burns SF, Broom DR, Miyashita M, Ueda C \& Stensel DJ (2006) Increased postprandial triacylglycerol concentrations following resistance exercise. Med Sci Sports Exerc 38, 527-533.

Burns SF, Corrie H, Holder E, Nightingale T \& Stensel DJ (2005) A single session of resistance exercise does not reduce postprandial lipaemia. J Sports Sci 23, 251-260.

Chapman J, Garvin AW, Ward A \& Cartee GD (2002) Unaltered insulin sensitivity after resistance exercise bout by postmenopausal women. Med Sci Sports Exerc 34, 936-941.

Coyle EF (2000) Physical activity as a metabolic stressor. Am J Clin Nutr 72, 512S-520S.

Essen-Gustavsson B \& Tesch PA (1990) Glycogen and triglyceride utilization in relation to muscle metabolic characteristics in men performing heavy-resistance exercise. Eur J Appl Physiol 61, $5-10$.
Fenicchia LM, Kanaley JA, Azevedo JL Jr, Miller CS, Weinstock RS, Carhart RL \& Ploutz-Snyder LL (2004) Influence of resistance exercise training on glucose control in women with type 2 diabetes. Metabolism 53, 284-289.

Ferguson MF, Alderson NL, Trost SG, Essig DA, Burke JR \& Durstine JL (1998) Effects of four different single exercise sessions on lipids, lipoproteins, and lipoprotein lipase. J Appl Physiol 85, 1169-1174

Fluckey JD, Hickey MS, Brambrink JK, Hart KK, Alexander K \& Craig BW (1994) Effects of resistance exercise on glucose tolerance in normal and glucose-intolerant subjects. J Appl Physiol 77, 1087-1092.

Gill JMR \& Hardman AE (2003) Exercise and postprandial lipid metabolism: an update on potential mechanisms and interactions with high-carbohydrate diets. J Nutr Biochem 14, 122-132.

Gill JMR, Herd SL \& Hardman AE (2002a) Moderate exercise and post-prandial metabolism: issues of dose-response. J Sport Sci 20, 961-967.

Gill JMR, Herd SL, Tsetsonis NV \& Hardman AE (2002b) Are the reductions in triacylglycerol and insulin levels after exercise related? Clin Sci 102, 223-231.

Gill JMR, Herd SL, Vora V \& Hardman AE (2003) Effects of a brisk walk on lipoprotein lipase activity and plasma triglyceride concentrations in the fasted and postprandial states. Eur J Appl Physiol 89, 184-190.

Gill JMR, Mees GP, Frayn KN \& Hardman AE (2001) Moderate exercise, postprandial lipaemia and triacylglycerol clearance. Eur J Clin Invest 31, 201-207.

Gill JMR, Murphy MH \& Hardman AE (1998) Postprandial lipemia: effects of intermittent versus continuous exercise. Med Sci Sports Exerc 30, 1515-1520.

Gotto AM Jr (1998) Triglyceride as a risk factor for coronary artery disease. Am J Cardiol 82, 22Q-25Q.

Hamilton MT, Etienne J, McClure WC, Pavey BS \& Holloway AK (1998) Role of local contractile activity and muscle fiber type on LPL regulation during exercise. Am J Physiol 275, E1016-E1022.

Jakicic JM, Clark K, Coleman E, Donnelly JE, Foreyt J, Melanson E, Volek J \& Volpe SL (2001) American College of Sports Medicine position stand. Appropriate intervention strategies for weight loss and prevention of weight regain for adults. Med Sci Sports Exerc 33, 2145-2156.

Jamurtas AZ, Koutedakis Y, Paschalis V, Tofas T, Yfanti C, Tsiokanos A, Koukoulis G, Kouretas D \& Loupos D (2004) The effects of a single bout of exercise on resting energy expenditure and respiratory exchange ratio. Eur J Appl Physiol 92, 393-398.

Kimber NE, Heigenhauser GJF, Spriet LL \& Dyck DJ (2003) Skeletal muscle fat and carbohydrate metabolism during recovery from glycogen-depleting exercise in humans. J Physiol 548, 919-927.

Kohrt WM, Bloomfield SA, Little KD, Nelson ME \& Yingling VR (2004) American College of Sports Medicine position stand. Physical activity and bone health. Med Sci Sports Exerc 36, 1985-1996.

Kolifa M, Petridou A \& Mougios V (2004) Effect of prior exercise on lipemia after a meal of moderate fat content. Eur J Clin Nutr $\mathbf{5 8}$, $1327-1335$

Koopman R, Manders RJ, Jonkers RA, Hul GB, Kuipers H \& van Loon LJ (2006) Intramyocellular lipid and glycogen content are reduced following resistance exercise in untrained healthy males. Eur J Appl Physiol 96, 525-534.

Koopman R, Manders RJ, Zorenc AH, Hul GB, Kuipers H, Keizer HA \& van Loon LJ (2005) A single session of resistance exercise enhances insulin sensitivity for at least $24 \mathrm{~h}$ in healthy men. Eur J Appl Physiol 94, 180-187.

Kraemer WJ, Adams K, Cafarelli E, et al. (2002) Position stand on the progression models in resistance training for healthy adults. Med Sci Sports Exerc 34, 364-380.

Lithell H, Cedermark M, Froberg J, Tesch P \& Karlsson J (1981) Increase of lipoprotein-lipase activity in skeletal muscle during 
heavy exercise. Relation to epinephrine secretion. Metabolism 30, $1130-1134$.

Lithell H, Schelle R, Vessby B \& Jacobs I (1984) Lipoproteins, lipoprotein lipase, and glycogen after prolonged physical activity. J Appl Physiol 57, 698-702.

Malkova D, Evans RD, Frayn KN, Humphreys SM, Jones PRM \& Hardman AE (2000) Prior exercise and postprandial substrate extraction across the human leg. Am J Physiol 279, E1020-E1028.

Malkova D, Hardman AE, Bowness RJ \& Macdonald IA (1999) The reduction in postprandial lipemia after exercise is independent of the relative contributions of fat and carbohydrate to energy metabolism during exercise. Metabolism 48, 245-251.

Melanson EL, Sharp TA, Seagle HM, Donahoo WT, Grunwald GK, Peters JC, Hamilton JT \& Hill JO (2002) Resistance and aerobic exercise have similar effects on 24-h nutrient oxidation. Med Sci Sports Exerc 34, 1793-1800.

Melby C, Scholl C, Edwards G \& Bullough R (1993) Effects of acute resistance exercise on postexercise energy expenditure and resting metabolic rate. J Appl Physiol 75, 1847-1853.

Miyashita M, Burns SF \& Stensel DJ (2006) Exercise and postprandial lipemia: effects of continuous compared with intermittent activity patterns. Am J Clin Nutr 83, 24-29.

Murphy MH, Nevill AM \& Hardman AE (2000) Different patterns of brisk walking are equally effective in decreasing postprandial lipemia. Int J Obes 24, 1303-1309.

Osterberg KL \& Melby C (2000) Effect of acute resistance exercise on postexercise oxygen consumption and resting metabolic rate in young women. Int J Sport Nutr Exerc Metab 10, 71-81.
Petitt DS, Arngrimsson SA \& Cureton KJ (2003) Effect of resistance exercise on postprandial lipemia. J Appl Physiol 94, 694-700.

Rhea MR, Alvar BA, Burkett LN \& Ball SD (2003) A meta-analysis to determine the dose response for strength development. Med Sci Sports Exerc 35, 456-464.

Shannon KA, Shannon RM, Clore JN, Gennings C, Warren BJ \& Potteiger JA (2005) Resistance exercise and postprandial lipemia: the dose effect of differing volumes of acute resistance exercise bouts. Metabolism 54, 756-763.

Sigal RJ, Kenny GP, Wasserman DH, Castaneda-Sceppa C \& White RD (2006) Physical activity/exercise and type 2 diabetes. A consensus statement from the American Diabetes Association. Diabetes Care 29, 1433-1438.

Slaughter MH, Lohman TG, Boileau RA, Horswill CA, Stillman RJ, Van Loan MD \& Bemben DA (1988) Skinfold equations for estimation of body fatness in children and youth. Human Biol 60, 709-723.

Tsetsonis NV \& Hardman AE (1996) Effects of low and moderate intensity treadmill walking on postprandial lipaemia in healthy young adults. Eur J Appl Physiol 73, 419-426.

Tsetsonis NV, Hardman AE \& Mastana SS (1997) Acute effects of exercise on postprandial lipemia: a comparative study in trained and untrained middle-aged women. Am J Clin Nutr $\mathbf{6 5}$, 525-533.

Vanhelder WP, Radomski MW, Goode RC \& Casey K (1985) Hormonal and metabolic response to three types of exercise of equal duration and external work output. Eur J Appl Physiol 54, $337-342$. 